\title{
First report of ageratum yellow vein virus infecting tomato in Vietnam
}

\author{
Hoseong Choi ${ }^{1,2} \cdot$ Yeonhwa Jo ${ }^{2} \cdot$ Phu-Tri Tran ${ }^{1} \cdot$ Kook-Hyung Kim ${ }^{1,2}$ (D)
}

Received: 29 October 2018 / Accepted: 10 May 2019/Published online: 29 May 2019

(C) Società Italiana di Patologia Vegetale (S.I.Pa.V.) 2019

Keywords Ageratum yellow vein virus $\cdot$ Begomovirus · Tomato $\cdot$ Vietnam

Geminiviruses have single-stranded DNA circular genomes and infect various crops such as tomato and pepper worldwide. Geminiviruses cause diverse disease symptoms such as mosaic, chlorosis, necrosis, malformation, and stunting (Andou et al. 2010; Fukuta et al. 2003; Jones et al. 2014). In 2017, we collected tomato leaf tissues showing symptoms such as yellowing, mosaic, and stunting from six different regions in Bao Loc city of Vietnam. We obtained and pooled leaf samples from at least three different farms for each region, isolated total RNAs from pooled tissues using RNeasy Microarray Tissue Mini kit (QIAGEN, CA, USA), and prepared six libraries for the tomato transcriptome analysis using the TruSeq RNA Library Preparation Kit V2 (Illumina, CA, USA). The libraries were analyzed by the paired-end method using the Illumina HiSeq 2000 platform (Macrogen, Korea). The obtained raw data were processed with Trinity software for the de novo assembly (Jo et al. 2016). Among the assembled contigs, one single contig (2570 nt) assembled from 312 reads from one library was matched to ageratum yellow vein virus (AYVV, KC172826; a monopartite begomovirus) with $94 \%$ sequence similarity. To confirm infection of AYVV in the collected sample, PCR was performed with two AYVV-specific primer sets designed based upon assembled contig sequence, i.e. 5'-CTGA TGTGCCCAAAGGTTGT-3' / 5'- GCCTGCTCCTTAGA CGCATA-3' and 5'-AGTGCCAAAGGGCCTTCTAT-3' / 5'-

Hoseong Choi

bioplanths@snu.ac.kr

$\triangle$ Kook-Hyung Kim

kookkim@snu.ac.kr

1 Department of Agricultural Biotechnology and Institute of Agriculture and Life Sciences, College of Agriculture and Life Sciences, Seoul National University, Seoul 08826, Republic of Korea

2 Plant Genomics and Breeding Institute, Seoul National University, Seoul 08826, Republic of Korea
TCACAGGTGGACAATATGCG-3', for amplification of full length DNA. The amplified fragment (2740 nt) was cloned into the pGEM T-easy vector (Promega, WI, USA) and sequenced. The sequence results revealed that the AYVV in Vietnam (MH750441) had 93\% sequence identity (2550/2747 nt) with ageratum yellow vein virus (AB100305.1). As judged from the NGS data, all tomato samples used in this study were also coinfected with tomato mosaic virus, tomato yellow leaf curl Kanchanaburi virus, and capsicum chlorosis virus. Therefore, we could not confirm that the symptoms observed such as yellowing, mosaic, and stunting on tomato leaves were caused by AYVV infection. Further efforts are needed to determine the extent of its detrimental effects in tomato. To our knowledge, this is the first report of AYVV infecting tomato in Vietnam.

Funding This work was funded by the Agenda Program (No. PJ014249032019), the Rural Development Administration (RDA), Republic of Korea.

\section{References}

Andou T, Yamaguchi A, Kawano S, Kawabe K, Ueda S, Onuki M (2010) Ageratum yellow vein virus isolated from tomato plants with leaf curl on Ishigaki Island, Okinawa, Japan. J Gen Plant Pathol 76:287291

Fukuta S, Kato S, Yoshida K, Mizukami Y, Ishida A, Ueda J, Kanbe M, Ishimoto Y (2003) Detection of tomato yellow leaf curl virus by loop-mediated isothermal amplification reaction. J Virol Methods 112:35-40

Jo Y, Choi H, Kim S-M, Kim S-L, Lee BC, Cho WK (2016) Integrated analyses using RNA-Seq data reveal viral genomes, single nucleotide variations, the phylogenetic relationship, and recombination for apple stem grooving virus. BMC Genomics 17:579

Jones JB, Zitter TA, Momol TM, Miller SA (2014) Compendium of tomato diseases and pests, 2nd edn. APS Press, St. Paul

Publisher's note Springer Nature remains neutral with regard to jurisdictional claims in published maps and institutional affiliations. 